Ann. Zootech., I975, 24 (2), 323-326.

\title{
ESTIMATION DE LA COMPOSITION CHIMIQUE DES CARCASSES DE JEUNES BOVINS MÂLES A PARTIR DE LA PROPORTION DE DÉPÔTS ADIPEUX D'UN MORCEAU MONOCOSTAL PRÉLEVÉ AU NIVEAU DE LA 11 ${ }^{\mathrm{e}}$ CÔTE
}

\author{
J. ROBELIN, Y. GEAY et C. BÉRANGER \\ Laboratoire de la Production de Viande, \\ Centre de Recherches de Clermont-Ferrand, I. N. R. A., \\ Theix, Saint-Genès Champanelle, 63110 Bicaumont.
}

\section{RÉSUMÉ}

Nous avons relié la composition chimique de la carcasse de 80 jeunes bovins mâles, au pourcentage de dépôts adipeux ou au pourcentage des différents composants chimiques d'un morceau monocostal (" I I ${ }^{\mathrm{e}}$ côte $\left."\right)$. Le pourcentage de dépôts adipeux de ce morceau est en très forte corrélation avec le pourcentage d'eau $(\mathrm{R}=-937)$, de lipides $(\mathrm{R}=0,952)$ et la valeur calorifique de la carcasse ( $R=0,948)$; la corrélation avec le pourcentage de protéines est plus faible $(R=-0,699)$. Les corrélations entre la composition chimique de la carcasse et la composition chimique de la I I $^{\mathbf{e}}$ côte ne sont pas significativement plus élevées que celles entre la composition chimique de la carcasse et le pourcentage de dépôts adipeux dans la i $\mathbf{e}^{\mathbf{e}}$ cote. Nous avons donc retenu ce dernier critère, pour l'estimation de la composition chimique des carcasses de jeunes bovins.

La dissection complète des carcasses de bovins ne peut être pratiquéc que sur un nombre limité d'animaux, parce qu'elle est longue et onéreuse. Geay et Beranger (ig69), ont proposé des relations permettant d'estimer la composition anatomique de la carcasse (proportions de dépôts adipeux, de muscles et d'os) à partir de celle d'un morceau monocostal prélevé au niveau de la I I $^{\mathrm{C}}$ côte. Cependant, l'estimation de la composition chimique des carcasses (pourcentages d'eau, dc matières grasses, de matic̀res minérales et de matières azotées), nécessite encore la dissection, le broyage et l'analyse chimique des différents tissus de celles-ci. Plusieurs auteurs ont déjà essayé de faire une estimation indirecte à partir de la composition chimique d'un morceau de la carcasse (Hopper, I944; Henninck et Englani), 1966; VAnce et al., 1971), mais leur estimation était limitée à la composition d'une partie de la carcasse (viande désosséc ou muscles parés). Nous avons donc essayé de relier la composition chimique de la carcasse entière à la composition anatomique ou chimique de la $\mathbf{I}^{\mathrm{e}}$ côte.

Nous avons utilisé pour cela 80 jeunes bovins mâles 4.37 lirisons, 2 I Charolais, Io Salcrs, 
I 2 Limousins) âgés de 9 à I 7 mois, et dont le poids de carcasse variait de I I 4 à $397 \mathrm{~kg}$. La demicarcasse droite a été disséquéc, et les tissus (os, muscles, dépôts adipcux) ont été broyés et analysés séparément; la teneur en matière sèche a été mesurée par lyophilisation; les matières grasses ont été extraites par le mélange chloroforme/méthanol $(2 / 1) ; 1$ la teneur en matières minérales a été mesurée après passage des échantillons au four à $600^{\circ} \mathrm{C}$; la valeur calorifique a été déterminée par calorimétric adiabatique. Enfin, la teneur en protéines a été calculée par diffẻrence. La I I $^{\mathrm{c}}$ côte a été prélevée sur la demi-carcasse gauche et disséquée d'après la méthode décrite par Y. Geay et C. Beranger (I969); les muscles et les dépôts adipeux ont ensuite été broyés et analysés ensemble.

A partir des résultats de dissection et d'analyse chimique, nous arons établi des relations entre les composants anatomiques et chimiques de la carcasse d'une part, et entre ceux-ci ct la composition de la i I e côte d'autre part. Dans un premier temps, ces relations ont été calculées à l'intérieur de chacune des races d'animaux. La comparaison des écluations de régression par analyse de covariance n'a pas permis de mettre en évidence des différences significatives cntre races dans les pentes ou les moyennes ajustées (tabl. r). Nous avons donc limité notre étude aux relations relatives à l'ensemble des animaux, considérant qu'elles étaient représentatives de chacune des races.

\section{TABLEAU I}

Estimation de la composition chimique de la carcasse à partir du pourcentage de dépóts adipeux dans la $11^{\circ}$ cote

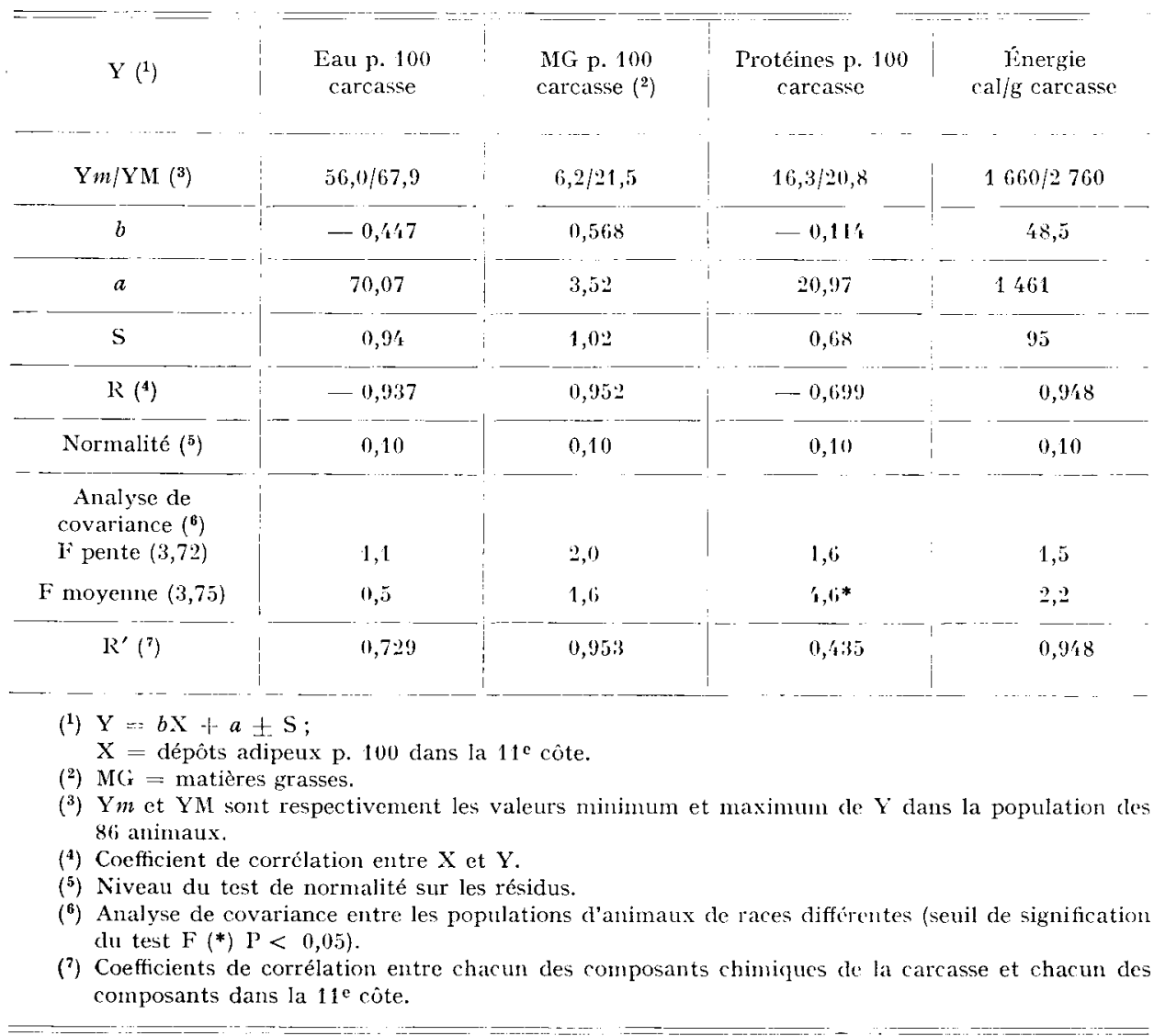


Les teneurs en eau et en matières grasses, ainsi que la valeur calorifique de la carcasse sont fortement reliées au pourcentage de dépôts adipcux de la $\mathbf{I}^{\mathbf{e}}{ }^{\mathrm{e}}$ cote ; les coefficients de corrélation sont égaux respectivement à - o,937, 0,952 et 0,948. Les cocfficients des équations de régression sont indiqués au tableau $r$

Ces liaisons étroites ne sont pas surprenantes, puisque la teneur cn matières grasses dans la carcasse est fortement dépendante de la proportion des dépôts adipeux $(R=0,964)$, qui est elle-même liée à la proportion de dépôts adipeux dans la I I ${ }^{\mathbf{C}}$ côte $(\mathrm{R}=0,942$, Geay et Beranger, I969). Or, la teneur en eau et la valeur calorifique de la carcasse sont étroitement dépendantes de la teneur en matières grasses de la carcasse $\left(R_{1}=0,956, R_{2}=0,992\right.$ respectivement). Ces résultats sont en accord avec les liaisons que différents auteurs ont mis en évidence chez les bovins, entre le pourcentage de dépôts adipeux dans la carcasse et le pourcentage de matières grasses dans sa viande désossée ( $\mathrm{R}=0,992$; CALlow, I948), ou entre le pourcentage de matières grasses et le pourcentage d'eau ( $\mathrm{R}=-0,998$; Callow, $19+7$; ReID et al., T968). La faible liaison que l'on observe entre le pourcentage de protéines dans la carcasse et le pourcentage de dépôts adipeux dans la I $\mathrm{I}^{\mathbf{e}}$ côte est surtout liée, scmble-t-il, à la variabilité extrêmement réduite de la tencur en matières azotées ( 6,3 à 20,8 p. I00), dans la population d'animaux que nous avons utilisée.

Les proportions des différents composants chimiques de la viande désossée de la I I e côte ne permettent pas de prédire avec une meilleure précision la composition chimique de l'ensemble de la carcasse, comme le montre la comparaison des coefficients de corrélation $R$ et $R^{\prime}$ au tableau $\mathbf{I}$.

A partir de la valeur des écarts types résiduels cles équations de régression mentionnées au tableau $\mathbf{I}$, on peut calculer la différence minimum qui peut être mise en évidence dans la composition chimique des carcasses entre deux lots d'animaux, si cette composition chimique a été estimée à partir de la proportion des dépôts adipeux de la I I e côte ; soit $\mathrm{X}$ la valeur de cette proportion, Y la valeur estimée de la teneur en l'un des composants chimiques de la carcasse $(\mathrm{Y}=b \mathrm{X}+a)$, S l'écart type résiduel de la régression, $\mathrm{V}$ la variance de $\mathrm{X}$ dans chacun des deux lots de $n$ animaux, la différence $\mathrm{D}=\left(\overline{\mathrm{Y}}_{1}-\overline{\mathrm{Y}}_{2}\right)$ entre les moyennes de Y sur chacun des deux lots n'est significative au seuil 0,05 que si elle dépasse la valeur $\mathrm{D}(0,0.5)$ :

$$
\mathrm{D}(0,05)=t(0,05) \sqrt{\frac{2}{n}\left(b^{2} \mathrm{~V}+\mathrm{S}^{2}\right)}
$$

Dans cette expression, $t(0,05)$ est la valeur du test " $t$ " de Student à $2 n-2$ degrés de liberté. Nous avons pu estimer la valeur de $\mathrm{V}$, variance de $\mathrm{X}$, sur une population de 34 animaux Frisons âgés de 15 mois : $V$ est voisin de 7 . Dans ces conditions, et d'après l'équation ( $\mathrm{I}$ ), la différence minimum qui peut être mise en évidence entre deux lots de ro animaux est égale à $\mathbf{I}, 4$ p. roo pour la proportion d'eau, I, $6 \mathrm{p}$. I oo pour celle des matières grasses, $0,6 \mathrm{p}$. Ioo pour celle des matières azotées et $\mathrm{I}_{42} \mathrm{cal} / \mathrm{g}$ pour la valcur calorifique. Ces valeurs sont relativement faibles par rapport aux différences de composition que l'on cherche à mettre en évidence entre lots de taurillons dans des expériences zootechniques.

Il est important de remarquer par ailleurs, que dans l'équation (1) la part de $S^{2}$ dans la variance de $\mathrm{Y},\left(b^{2} \mathrm{~V}+\mathrm{S}^{2}\right)$, ne dépasse jamais $5^{\circ} \mathrm{p}$. Ioo. Iin particulier, dans le cas des matières grasses, et d'après les valeurs de $b$ et $S$ indiquées au tableau $I, S^{2}$ ne représente que $3 \mathrm{I}, 5 \mathrm{p}$. гоo de la variance de $\mathrm{Y}$. La valeur de la différence $\mathrm{D}(0,05)$ dépend donc pour une large part de $V$, c'est-à-dire de l'homogénéité des lots d'animaux.

La proportion de dépôts adipeux de la I I $^{\mathrm{e}}$ côte, facile à mesurer, semble donc être un bon critère d'estimation de la composition chimique de la carcasse des jeunes bovins. Cette première analyse des résultats est prometteuse ; il est nécessaire de l'approfondir, en utilisant notamment les poids des composants au lieu des pourcentages qui ne permettent pas cle tenir compte de la variabilité du poids de carcasse, et en associant au poids des dépôts adipeux de la I I e côte, d'autres critères d'estimation facilement mesurables : poids de carcasse, poids des dépôts adipeux périrénaux, poids des os cánons... 


\title{
SUMMARY
}

\author{
PREDICTION OF CHEMICAI, COMPOSITION OF YOUNG BULI, CARCASSES, \\ FROM THE PERCENTAGE OF FAT DEPOSITS OF THE, IITH RIB-JOINT
}

The chemical composition of 80 young bull carcasses was related to the percentage of dissectable fat deposits, or the chemical composition of the i th rib-joint.

The percentage of dissectable fat in the joint was highly correlated with the percentage of water $(\mathrm{R}=-0.937)$. Iipids $\left(\mathrm{R}=0.95^{2}\right)$ and the caloric value of the carcass $(\mathrm{R}=0.948)$. The correlation with the percentage of protein was lower $(\mathrm{R}=-0.699)$. As the correlations between chemical composition of the carcass and chemical composition of the I th rib are not significantly higher than those between chemical composition of the carcass and percentage of dissectable fat in the rib, we have therefore chosen the latter as a predictor of the chemical composition of the carcass.

\section{RÉFÉRFNCES BIBIIOGRAPHIQUES}

Callow E. H., I947. Comparative studies of meat. I. The chemical composition of fatty and muscular tissue in relation to growth and fattening. J. A gric. Sci., 37, I $13-$ I 29.

Callow E. H., I948. Comparative studies of meat. II. The changes in the carcass during growth and fattening, and their relation to the chemical composition of the fatty and muscular tissues. $J$. A gric. Sci., 38, I 74-I 99 .

Geay Y., Beranger C., rg69. Estimation de la composition de la carcasse de jeunes bovins à partir de la composition d'un molceau monocostal au niveau de la i c' côte. Ann. Zootech., 18, 65-77.

HENANCK W. H., ENGLAND D. C., 1960. A method of estimating the percentage of protein and fat in the edille portion of stecr carcass. J. Anim. Sci., 19, I I9I.

Hopper T. H., I 944. Methods of estimating the physical and chemical composition of cattle. J.Agric. Res., 68, 239. Cité par GeaY' et BERAnGER (1969).

Reid J. T., Bensadoun A., Bult, L. S., Burton J. H., Gleeson P. A., Han I. K., Joo Y. D., Johnson 1). E., McManus W. R., Paladines O. L., Stroud J. W., Trrrell H. li, Van NieKERK B. D. H., Wellingtos G. H., WoOD J. I), I968. Changes in body composition and meat characteristics accompanying growth of animals. Proc. Cornell Conf. Feed. Manuf., 18-37.

Vance R. D., Ockliman I. W., Cahili, V. R., Plimpton R. F., ig7i. Chemical composition as related to selected measurements used in beef carcass evaluation. J. A nim. Sci., 33, 744-49.

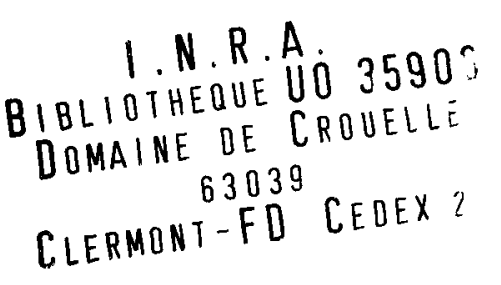

